\title{
Carcinoma basoescamoso: Reporte de un caso con exposición crónica a arsénico ambiental*
}

\author{
Drs. CONSUELO CÁRDENAS D. ${ }^{1}$, CLAUDIA SALOMONE B. ${ }^{1}$, FRANCISCO DOMÍNGUEZ C. ${ }^{2}$, \\ Int. FELIPE CICHERO Z. ${ }^{5}$, DRS. PABLO MUÑOZ S. ${ }^{3}$, SERGIO GONZÁLEZ B. ${ }^{4}$
}

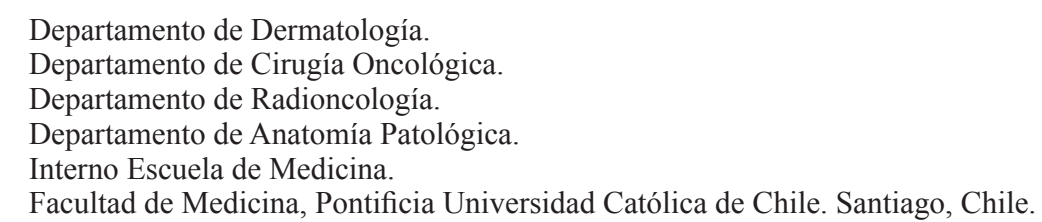

\section{Abstract \\ Basosquamous carcinoma: A case report with chronic environmental arsenic exposition}

Basosquamous carcinoma is a relatively rare cutaneous tumour that shares some characteristics of both basal cell and squamous cell carcinoma. Currently most dermatologists consider that it represents a subtype of basal cell carcinoma with a more aggressive behaviour. The clinical presentation is non-specific and in general the diagnosis is made after the histopathological studies. The prevalence is higher in male patients, during the seventh decade and it tends to appear on sun exposed areas. Many authors compare its behaviour with squamous cell carcinoma. We present the case of a 44 years old male patient with a history of chronic arsenic exposure that presented a firm tumor in the left inguinal region and the biopsy showed basosquamous carcinoma with lymphatic involvement. This case shows an atypical presentation because of its localization and the appearance 20 years before the mean age reported in the literature. This could be explained by chronic environmental arsenic exposure, a well known risk factor for the development of cutaneous tumours.

Key words: Basosquamous carcinoma, basal cell carcinoma, arsenic.

\section{Resumen}

El carcinoma basoescamoso es una neoplasia cutánea relativamente rara que reúne características de carcinoma basocelular y espinocelular. Actualmente la mayoría de los dermatólogos reconocen que este es un subtipo del carcinoma basocelular con comportamiento muchísimo más agresivo. Su presentación clínica es inespecífica y en general su diagnóstico se realiza sólo después de la biopsia. Predomina en pacientes de sexo masculino durante la séptima década de la vida y tiende a aparecer en regiones fotoexpuestas. Muchos autores igualan su comportamiento al del carcinoma espinocelular. Se presenta el caso de un paciente, de sexo masculino, de 44 años de edad, con antecedente de exposición a arsénico medioambiental crónica, que presentó aumento de volumen, duro en la región inguinal izquierda, cuya biopsia fue compatible con carcinoma basoescamoso con compromiso linfático. El caso de nuestro paciente muestra una presentación atípica por su localización y por la aparición 20 años antes de la media de edad reportada en la literatura. Esto podría ser explicado por la exposición crónica a arsénico, conocido factor de riesgo para carcinogénesis cutánea.

Palabras clave: Carcinoma basoescamoso, carcinoma basocelular, arsénico.

*Recibido el 15 de Octubre de 2009 y aceptado para publicación el 23 de Noviembre de 2009.

Correspondencia: Dra. Consuelo Cárdenas D.

Av. Vicuña Mackenna 4686, Santiago, Chile. Fax: (56 2) 5529974.

E-mail: cpcarden@gmail.com 


\section{Introducción}

El carcinoma basoescamoso es una neoplasia cutánea relativamente poco frecuente $(1,5$ a $2,7 \%$ de los cánceres de piel), que reúne características de carcinoma basocelular y espinocelular ${ }^{1,2}$. Su definición en la literatura es poco clara ya que se ha usado para nombrar distintos tipos de entidades patológicas, como la concomitancia de un carcinoma basocelular con un espinocelular, la queratinización de un carcinoma basocelular, o la diferenciación de un tipo de tumor hacia el otro ${ }^{3}$. Hoy en día, la mayoría de los autores reconocen que el carcinoma basoescamoso es un subtipo de carcinoma basocelular con comportamiento significativamente más agresivo ${ }^{4}$.

Su presentación clínica es poco característica, en general se llega al diagnóstico sólo después de la biopsia. Los datos recolectados en varias series de casos muestran un predominio de este tumor en pacientes de sexo masculino y alrededor de la séptima década de $v^{1} a^{1,2}$. La localización de estos tumores es similar a la de los otros tipos de carcinomas basocelulares. La mayoría de las lesiones aparecen en la región de la cabeza y el cuello $(80 \%)$, siendo la zona centro facial y perinasal las localizaciones más comunes $(30 \%)$. Sin embargo, el tumor puede aparecer también en el tronco y las extremidades ${ }^{4,5}$.

Muchos autores refieren que el carcinoma basoescamoso tiene peor pronóstico y mayor incidencia de recurrencia y metástasis que el carcinoma basocelular y algunos autores igualan su comportamiento al del carcinoma espinocelular ${ }^{1,4}$. Se han descrito tasas de recurrencia entre $12 \%$ y un $50 \%$ para los tumores basoescamosos de cabeza y cuello, y se han calculado tasas de metástasis entre un 5\% y hasta un 7,4\% en seguimientos de hasta 20 años $^{2,4,6}$.

No está bien establecido el mejor tratamiento para el carcinoma basoescamoso. Actualmente, el tratamiento estándar, incluye la resección amplia asegurándose márgenes negativos, evaluación del compromiso nodal y metástasis a distancia y seguimiento cuidadoso 5 . Se ha usado quimioterapia adyuvante con 5-fluorouracilo y radioterapia, pero no existen datos suficientes para comprobar su eficacia ${ }^{7}$.

\section{Caso clínico}

Paciente de 44 años, de sexo masculino, con antecedentes de resección, 3 años antes de consulta actual, de una lesión plantar izquierda cuya biopsia era compatible con un carcinoma espinocelular in situ, multifocal, con componente dérmico de carcinoma basocelular morfeiforme, realizada en otro centro. Vivió en Antofagasta, zona con altas concentracio- nes de arsénico medioambiental, durante aproximadamente 25 años. El cuadro actual se presenta con 7 meses de evolución del crecimiento progresivo de una masa indurada en la zona inguinal izquierda. Un mes antes de la consulta presentó ulceración de esta masa, sin dolor, secreción, ni otros signos inflamatorios. El paciente no presentaba compromiso del estado general, fiebre ni baja de peso.

Al examen físico se evidenció en la región inguinal izquierda un tumor duro de $3 \times 3 \mathrm{~cm}$, adherido a la piel circundante, mal definido. El tumor presentaba una úlcera central de fondo sucio, sin mal olor (Figura 1). No se palparon adenopatías. Se realizó cultivo de la lesión que resultó positivo para Enterobacter Aerogenes, indicándose tratamiento con Ciprofloxacino. La ecografía de partes blandas mostró una masa infiltrante de aspecto neoplásico en región anterior de la raíz del muslo izquierdo asociada a adenopatías adyacentes e inguinales ipsilaterales (Figura 2).

La biopsia incisional mostró tumor dermohipodérmico, ulcerado, infiltrativo, compuesto por células basaloides de núcleos ovoideos, nucléolo visible, citoplasma eosinófilo, dispuestas en nidos pequeños y medianos, focalmente continuas con la epidermis suprayacente, en partes con empalizada y fisuras periféricas y numerosas perlas córneas centrales; el estroma era desmoplástico denso. El estudio inmunohistoquímico mostró reacción positiva para p63 y reacción negativa para CEA, EMA y BRST-2. Todos estos hallazgos fueron compatibles con un carcinoma basoescamoso, ulcerado, infiltrante en dermis reticular (Figura 3).

La resección amplia de la lesión y de las masas sugerentes de adenopatías mostró carcinoma basoescamoso, ulcerado, infiltrante en hipodermis con compromiso del borde profundo de la muestra, invasión vascular y metástasis del carcinoma en los dos linfonodos examinados.

Se realizaron estudios de diseminación, que mostraron 2 adenopatías en cadena ilíaca externa izquierda, de 13 y $14 \mathrm{~mm}$ (Figura 4) en el TAC de abdomen y pelvis. Se realizó además un cintigrama óseo y una radiografía de tórax cuyos resultados fueron normales.

En un segundo tiempo quirúrgico se realizó una resección cutánea ampliada de la región inguinofemoral izquierda y una disección ilio-femoral izquierda. La biopsia del tejido resecado no mostró tumor primario residual en piel, pero evidenció metástasis en 2 de 10 ganglios linfáticos femorales, una invasión vascular venosa periganglionar y una adenopatía ilíaca externa positiva (de 8 ganglios linfáticos ilíacos extirpados). En resumen, el paciente presentó 5 metástasis ganglionares linfáticas, 4 de 12 femorales extirpadas y 1 de 8 ilíacas externas 


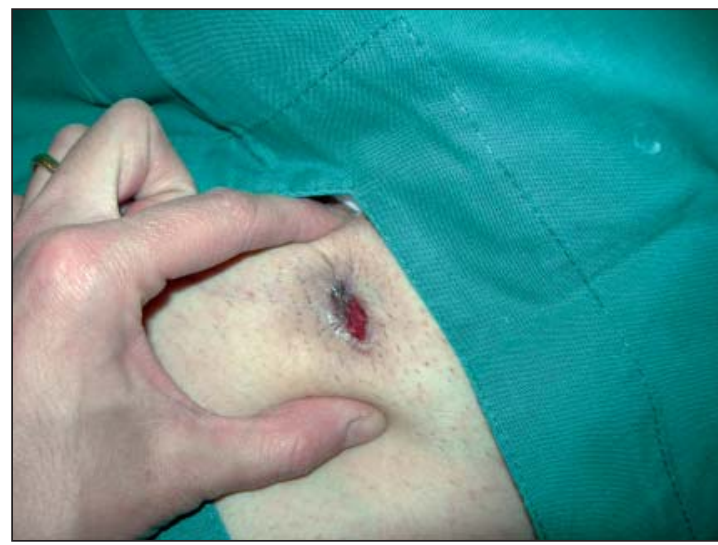

Figura 1. Tumor de $3 \times 3 \mathrm{~cm}$, adherido a la piel, con úlcera central de fondo sucio.

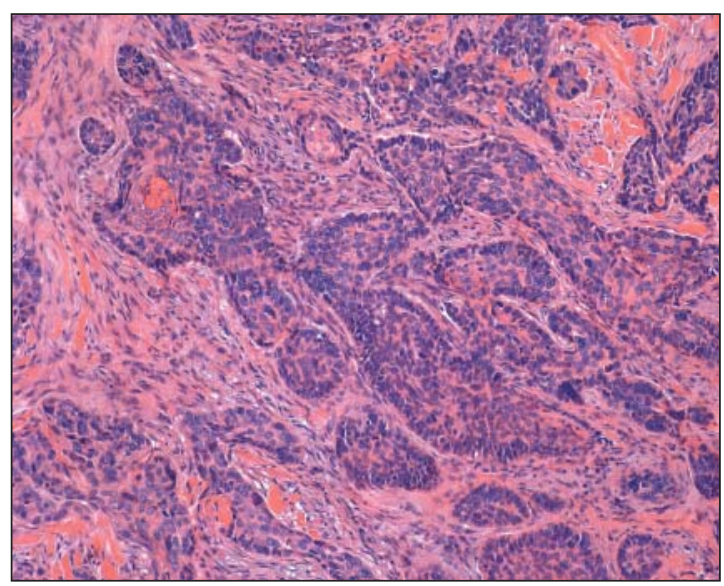

Figura 3. Carcinoma basoescamoso, ulcerado, infiltrante en dermis reticular con perlas córneas y empalizadas. HE, 100x.

extirpadas. Se presentó el caso a discusión en reunión multidisciplinaria de oncología, planteándose el uso de radioterapia local y regional dado un alto riesgo de recurrencia, opción que fue rechazada por el paciente.

\section{Discusión}

El carcinoma basoescamoso es un cáncer de piel de baja incidencia, pero de gran importancia diagnóstica, ya que su comportamiento puede ser similar al carcinoma espinocelular, llegando incluso a superarlo en la incidencia de recurrencias y metástasis según algunos estudios ${ }^{2,7,8}$. La gran heterogeneidad de la presentación clínica del carcinoma basoesca-

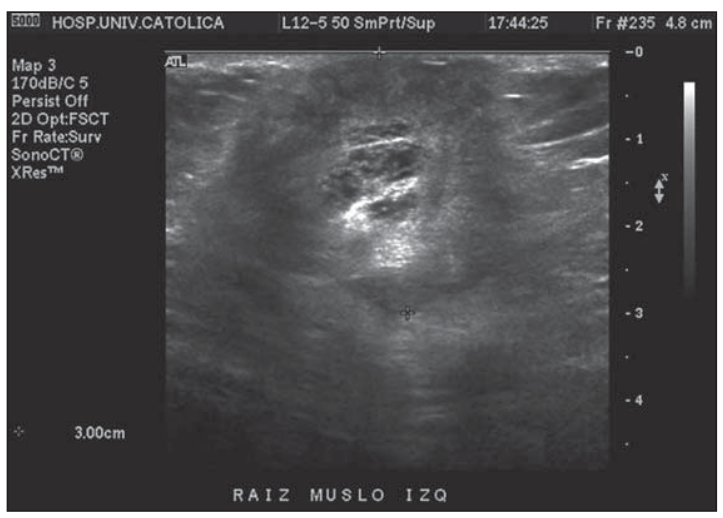

Figura 2. Masa infiltrante lobulada de $3,1 \times 3,3 \mathrm{~cm}$, que compromete tejido adiposo subcutáneo y piel con áreas quísticas centrales.

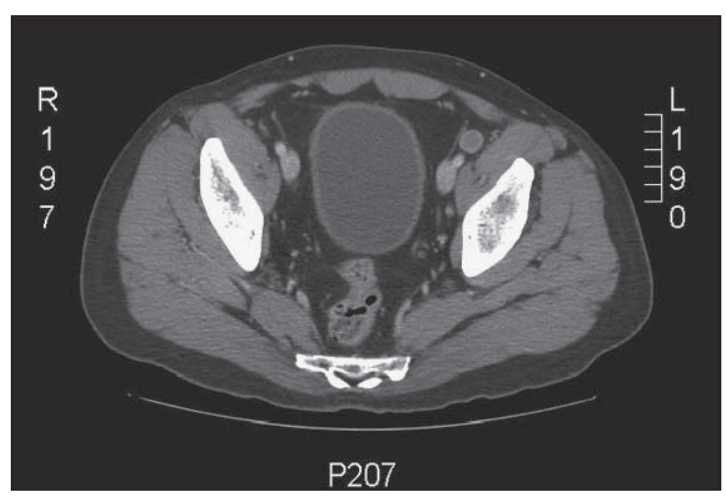

Figura 4. Cambios postquirúrgicos en región inguinal izquierda. Adenopatías ilíacas izquierdas.

moso hace que el diagnóstico se realice mediante la biopsia, siendo necesario un acucioso trabajo anatomopatológico para distinguir esta entidad de otros tipos de carcinomas.

La definición histológica de este tumor es confusa. Una extensa revisión realizada este año, propone definirlo como un subtipo de carcinoma basocelular con crecimiento infiltrativo, que muestra áreas de carcinoma basocelular y espinocelular, con o sin zona de transición y con un estroma colagenizado rico en fibroblastos ${ }^{4}$.

Las características clínicas son muy variadas, pero existe una tendencia marcada a desarrollarse en la zona de cabeza y cuello en hombres alrededor de la séptima década de vida. El caso de nuestro paciente muestra por una parte, una presentación 
atípica por la localización en zona no fotoexpuesta y por la aparición casi 20 años antes de la media de edad reportada en la literatura ${ }^{1}$, y por otra parte, una lesión previa en la planta de características histopatológicas semejantes: un carcinoma espinocelular in situ y un basocelular invasor, ambas lesiones inusuales en la planta en un paciente de esta edad. Ambos hechos podrían explicarse por una exposición crónica al arsénico, ya que el paciente vivió por más de 25 años en Antofagasta, zona donde se han demostrado altos niveles de contaminación con este metal en el agua potable desde al menos la década del $70^{9}$. La exposición crónica a arsénico se asocia a la inducción de lesiones neoplásicas, con una relación que es dosis-dependiente entre los niveles de arsénico en el agua potable y los carcinomas cutáneos. Los cánceres de piel inducidos por arsénico más comunes son la enfermedad de Bowen, el carcinoma basocelular y el carcinoma espinocelular. Estas neoplasias, cuando son inducidas por el arsénico, tienen la característica de aparecer en áreas no fotoexpuestas del cuerpo, presentar múltiples lesiones y mayor probabilidad de recidiva ${ }^{10}$. En el caso que estamos presentando, nuestro paciente tuvo dos neoplasias cutáneas, un carcinoma espinocelular y un carcinoma basoescamoso, en zonas no fotoexpuestas. Sin embargo, la hipótesis de que estos tumores hayan sido inducidos por arsénico sólo se sustenta en evidencia epidemiológica, ya que no tuvimos la oportunidad de demostrar arsenicismo crónico en el paciente.

El tratamiento del carcinoma basoescamoso no ha sido bien estudiado, siendo la resección quirúrgica del tumor la terapia actual de elección. La gran incidencia de metástasis linfonodales en el momento de presentación de esta neoplasia sugiere el uso de la técnica del linfonodo centinela como herramienta para su detección precoz cuando las linfoadenopatías no son palpables, a diferencia del caso de nuestro paciente $^{2}$.

Se han descrito factores de riesgo de mayor recurrencia del carcinoma basoescamoso. Los más significativos son márgenes quirúrgicos positivos, invasión linfática, invasión perineural y sexo masculino $^{1}$. Nuestro paciente presenta al menos dos de estas condiciones, por lo que es fundamental un seguimiento cercano para detectar las recidivas precozmente. Aunque no se ha demostrado la eficacia de la quimioterapia y la radioterapia en el tratamiento del carcinoma basoescamoso, la sensibilidad a estos agentes de otros carcinomas cutáneos similares, hacen razonable que sean considerados como armas terapéuticas, en especial en pacientes con alto riesgo de recidiva como el presente caso.

\section{Referencias}

1. Martin C, Edwards J, Cawte G, Sewell L, McMasters M. Basosquamous carcinoma: analysis of prognostic factors influencing recurrence. Cancer 2000; 88: 1365 1369.

2. Bowman H, Ratz L, Knoepp G, Barnes J, Finley M. Basosquamous carcinoma. Dermatol Surg 2003; 29: 830-832.

3. Maloney M. What is basosquamous carcinoma? Dermatol Surg 2000; 26: 505-506.

4. García C, Poletti E, Crowson N. Basosquamous carcinoma. J Am Acad Dermatol 2009; 60: 137-143.

5. Costantino D, Loweb L, Brown D. Basosquamous carcinoma-an under-recognized, high-risk cutaneous neoplasm: Case study and review of the literature. Journal of Plastic, Reconstructive \& Aesthetic Surgery 2006; 59: 424-428.

6. López de Faria J, Núñez P. Basosquamous cell carcinoma of the skin with metastases. Histopathology 1988; 12: 85-94.

7. Johnson B, Moore P, Goepel J, Slater D. Basosquamous carcinoma, a wolf in sheep's clothing? Report of 3 cases. Postgrad Med J 1989; 65: 750-751.

8. Petri W, Zoldos J, Wilson T. Surgical management of basosquamous carcinoma with perineural invasion. J Oral Maxillofac Surg 1995; 53: 951-954.

9. Borgono J, Vicent P, Venturino H, Infante A. Arsenic in the drinking water of the city of Antofagasta: epidemiological and clinical study before and after the installation of a treatment plant. Environ Health Perspect 1977; 19: 103-105.

10. Yu H, Liao W, Chai C. Arsenic carcinogenesis in the skin. J Biomed Sci 2006; 13: 657-666. 